\title{
Diacronie
}

Studi di Storia Contemporanea

$N^{\circ} 20,4 \mid 2014$

II diritto militante

\section{La cooperazione di consumo nel Monfalconese nella seconda metà del Novecento}

Dalla Cooperativa Consumo Lavoratori del Monfalconese alla Coop Consumatori Nordest

\section{Enrico Bullian}

\section{(2) OpenEdition \\ Journals}

Edizione digitale

URL: http://journals.openedition.org/diacronie/1796

DOI: 10.4000/diacronie. 1796

ISSN: 2038-0925

Editore

Association culturelle Diacronie

Notizia bibliografica digitale

Enrico Bullian, « La cooperazione di consumo nel Monfalconese nella seconda metà del Novecento », Diacronie [Online], $N^{\circ}$ 20, 4 | 2014, documento 15, Messo online il 01 décembre 2014, consultato il 01 mai 2019. URL : http://journals.openedition.org/diacronie/1796 ; DOI : 10.4000/diacronie.1796 


\section{Diacronie}

N. 20 | 4|2014 Il diritto militante

15/

\section{La cooperazione di consumo nel}

Monfalconese nella seconda metà del

Novecento

\section{Dalla Cooperativa Consumo Lavoratori del Monfalconese alla Coop Consumatori Nordest *}

Enrico BULLIAN *

Lo studio sull'evoluzione della cooperazione di consumo nel Monfalconese dal secondo dopoguerra restituisce un quadro mai ricostruito dalla storiografia, perlomeno su una dimensione locale. Si analizzano da una parte la crescita delle strutture aziendali dall'originaria Cooperativa Consumo Lavoratori del Monfalconese (1945), alla Coop Consumatori Friuli (1985) e infine alla Coop Consumatori Nordest (1995); dall'altra i passaggi dai "negozi di paese" al Discount di Monfalcone degli anni Settanta fino alla successiva sostituzione con un rinnovato Supercoop. In particolare la costituzione di uno dei primi Discount a livello nazionale rappresentò per il territorio e per la cooperazione di consumo un'esperienza sperimentale fondante, resa possibile dai collegamenti con gli enti locali e le organizzazioni politiche e sindacali di allora.

\section{La rinascita della cooperazione nel secondo dopoguerra e la creazione della prima rete di negozi}

a storia della cooperazione - se considerata come associazione spontanea di soggetti che si prefiggono delle finalità comuni attraverso decisioni condivise a proprio beneficio - si potrebbe far iniziare con l'avvento della specie umana. Nel mondo contemporaneo ha raggiunto una maggior 
strutturazione, inserendosi in molti settori dell'economia e della vita sociale ${ }^{1}$. In questa ricerca interessa in particolare l'evoluzione della cooperazione di consumo nel mandamento Monfalconese a partire dal secondo dopoguerra ${ }^{2}$.

I combattimenti e i bombardamenti avvenuti nel corso dell'ultimo conflitto bellico segnarono profondamente il territorio Monfalconese, che fu pesantemente condizionato dalla "costruzione" del nuovo confine con la Jugoslavia, che rappresentava anche la separazione fra due mondi. Il Monfalconese subì le conseguenze della sconfitta dell'Italia e rientrò fra le aree contese dalla Repubblica socialista guidata da Tito, che intendeva annetterle al proprio territorio. Tuttavia la Liberazione dai nazifascisti permise una nuova stagione di fioritura per la cooperazione a livello nazionale e anche nel Monfalconese.

La ricerca storica non si è ancora mai focalizzata sull'evoluzione della cooperazione di consumo nel Monfalconese, se non attraverso passaggi molto sintetici all'interno di pubblicazioni che si soffermavano sull'Italia nord orientale, come nel caso di Franco Bojardi:

La Cooperativa Consumo Lavoratori del Monfalconese nasce nel novembre del 1945, frutto della tradizione e della storia cooperativa già esistente a Monfalcone

\footnotetext{
* Il saggio - pubblicato su due numeri della rivista - è frutto della borsa di ricerca dedicata allo studio di alcune aziende del Monfalconese. La borsa è stata gestita dal Consorzio per l'AREA di ricerca scientifica e tecnologica di Trieste (con cofinanziamento del Fondo Sociale Europeo), mentre il Dipartimento di Studi Umanistici dell’Università degli Studi di Trieste è stato la struttura ospitante dell'attività di ricerca, in collaborazione con le 4 imprese partner (TreCoFer, Nuovo Arsenale Cartubi, Strato e Coop Consumatori Nordest).
}

1 Nel 1924 Sandro Pertini, nell'introduzione della propria tesi di laurea dedicata alla cooperazione, sosteneva: «Se rendiamo il faticoso cammino percorso da l'umanità, possiamo volendo - trovare i germi delle odierne società cooperative in antichissime istituzioni greche romane ed anche ebraiche - ed in altre meno remote apparse nell'età di mezzo: ma noi riteniamo oziosa una tale indagine storica, perché se si possono trovare delle analogie tra le cooperative odierne ed alcune antiche istituzioni, è tuttavia evidente la differenza del principio economico, sul quale esse riposano». PERTINI, Sandro, La cooperazione, Savona, Associazione per lo studio del mutualismo e dell'economia solidale, 2013, p. 3. Per quanto attiene l'età contemporanea, la storiografia ha convenzionalmente individuato delle cesure dalle quali si sviluppano le attuali cooperative. A livello internazionale l'origine storica è stata individuata nella definizione degli obiettivi dell'associazione degli Equitables pioneers of Rochdale del 24 ottobre 1844, mentre a livello nazionale il 4 ottobre 1854 apriva il primo Magazzino di previdenza (un distributorio sociale) della Società generale degli operai di Torino. Cfr. BOJARDI, Franco (a cura di), Dalla cooperazione di Consumo alla Cooperazione dei Consumatori nell'Italia del Nord-Est, Reggio Emilia, Coop Consumatori Nordest, 1994, pp. 5-6; ZAMAGNI, Vera, BATTILANI, Patrizia, CASALI, Antonio, La cooperazione di consumo in Italia. Centocinquant'anni della Coop consumatori: dal primo spaccio a leader della moderna distribuzione, Bologna, Il Mulino, 2004, pp. 5-11.

${ }^{2} \mathrm{Al}$ mandamento Monfalconese - che conta circa 70.000 abitanti - appartengono i Comuni di Monfalcone, Ronchi dei Legionari, Staranzano, San Canzian d'Isonzo, Turriaco, San Pier d'Isonzo, Fogliano Redipuglia, Sagrado e Doberdò del Lago. 
prima dell'avvento del fascismo e sulla spinta delle esigenze di tutela dei salari della classe operaia locale.

I primi due negozi sono ubicati uno all'interno della città e l'altro nelle vicinanze dello stabilimento C.R.D.A. (Cantieri riuniti dell'Adriatico) che tanta parte ha della storia della città di Monfalcone.

La prima quota di adesione fu fissata in L. 500 con la possibilità di versamenti rateali di L. 50 al mese.

In breve tempo la piccola cooperativa superò le 5.000 adesioni e su questa spinta si sviluppò con una rete di negozi in alcuni comuni del mandamento monfalconese e della bassa friulana ed anche con un negozio di tessuti ed abbigliamento a Cormons.

In quest'opera si sono senz'altro distinti i primi presidenti Pangher, De Lise ed inoltre Tonizzo rimasto alla guida della cooperativa sino al 1977.

Ed ancora vanno ricordati alcuni cooperatori come Mauchigna di Ronchi dei Legionari, Benfatto di Turriaco, Goia e Gallovics di Monfalcone3.

Cercando di approfondire maggiormente tale ricostruzione, si può iniziare la riflessione contestualizzando la realtà del mandamento Monfalconese, ovvero di un territorio nel quale non era stata ancora definita la collocazione nazionale. Qui il mondo cooperativo rinacque - come ricorda Bojardi - già a partire dal 1945. Lo dimostra anche lo Statuto sociale della Cooperativa Consumo dei Lavoratori del Monfalconese $(C C L M)^{4}$, che rappresentò la prima tappa della costruzione della rete di negozi che rimase operante fino alla seconda metà degli anni Settanta. È interessante notare che l'avvio di questa fase era precedente al riconoscimento della funzione sociale della cooperazione nella Costituzione (art. 45), addirittura antecedente alla stessa elezione dell'Assemblea Costituente (2 giugno 1946). Il primo Statuto della CCLM risentiva del contesto di allora e del clima di incertezza legato al destino della Venezia Giulia. Procedendo con ordine, l'impostazione dello Statuto era già chiara nel suo art. 1, dove si stabiliva che la società cooperativa «è costituita in Monfalcone tra operai, impiegati e intellettuali» e che «potrà aprire filiali nei comuni della Venezia Giulia». All'art. 5 si

3 BOJARDI, Franco (a cura di), op. cit., pp. 42, 46.

4 Archivio Storico Sindacale "Sergio Parenzan" della CGIL di Gorizia (d'ora in avanti, A. CGIL), Lettura Record: n. file 312/1/1, Statuto sociale della Cooperativa Consumo dei Lavoratori del Monfalconese C.C.L.M. Società cooperativa a responsabilità limitata Monfalcone, Monfalcone, Stabilimento Tipografico Moderno, 1945. In occasione di questa prima citazione della documentazione proveniente dall'A. CGIL, si specifica il significato delle cifre della Lettura Record, che seguirà la medesima impostazione nelle successive note: $n$. file 312/1/2= faldone $n$. 312; cartella/fascicolo n. 1; documento n. 2. Nell'Archivio sono contenuti importanti documenti sulla cooperazione di consumo nel Monfalconese in particolare nei seguenti Record: faldone $\mathrm{n}$. 312; cartella/fascicolo nn. 1-2 (quasi tutti $\mathrm{i}$ documenti contenuti in entrambe le cartelle/fascicoli). 
affermava che i soci dovevano risiedere nella Venezia Giulia, area che tuttavia in quel momento non era semplice identificare in maniera condivisa e che fu separata prima dal confine tra la zona A e la zona B (1946-1947), poi da quello tra l'Italia, il Territorio Libero di Trieste e la Jugoslavia (1947-1954) e, infine, tra l'Italia e la Jugoslavia (dopo il 1954). Qui riaffiora la complessità della storia del confine orientale, dove fino al 1947 operava il Partito Comunista della Venezia Giulia, che sosteneva per questo territorio (e quindi anche per il Monfalconese) l'ingresso nella Repubblica socialista Jugoslava. Quindi, probabilmente in considerazione di tale incertezza, il primo statuto della CCLM - in attesa della definizione dei confini - si riferiva alla Venezia Giulia senza citare la realtà statuale. Inoltre il perimetro dei soci era limitato a «operai, impiegati e intellettuali». Nello Statuto si ritrovava la consapevolezza di aver acquisito la libertà e per la gestione della cooperativa si creava un percorso democratico, che per molti cittadini fu sicuramente un'occasione per esercitare concretamente forme di diritto di voto e di partecipazione alle decisioni (art. 20, le deliberazioni dell'assemblea dei soci «sono prese a maggioranza assoluta di voti dei soci presenti»5). Si segnala l'art. 17, che sanciva un principio fondamentale della cooperazione che continua a differenziarla da una società di capitali ( «Il socio ha diritto ad un solo voto nell'assemblea, qualsiasi sia il numero delle azioni in suo possesso») e l'art. 36 sulla ripartizione degli utili netti, che vedeva il 10 per cento destinato per l'«assistenza ai soci ed alle famiglie dei Caduti per la causa della liberazione». All'art. 38 si affermava che «tutte le cariche sociali sono normalmente gratuite».

Questi ultimi due articoli non vennero riproposti nel nuovo statuto approvato nel $1963^{6}$. Da una parte si prevedeva di utilizzare i residui attivi di bilancio per più generici scopi di assistenza sanitaria, culturale, ricreativa e mutualistica a favore dei soci e delle famiglie (art. 22). Dall'altra, ai membri del Consiglio di Amministrazione (CdA) - che a tempo pieno di occupavano della cooperativa - spettava un compenso (art. 30). Lo Statuto del 1963 lasciava intravedere una situazione in espansione rispetto al 1945: il numero minimo di soci passava da 5 a 50 (art. 4); i componenti del CdA aumentavano da 5 a un numero variabile fra 7 e 17 (art. 30); veniva prevista la possibilità di svolgere assemblee nelle località sedi di negozi per favorire la partecipazione dei soci (art. 29); si istituiva un Comitato consultivo di spaccio (art. 41). All'art. 1 la CCLM aderiva alla "Lega Nazionale delle Cooperative e Mutue" e ai collegati organismi regionali o provinciali, riconoscendosi nell'organo ufficiale di stampa, il giornale la «Cooperazione

5 Il titolo IV (artt. 19-34) definiva gli organi sociali e le rispettive competenze.

${ }^{6}$ A. CGIL, Lettura Record: n. file 312/1/3, Cooperativa Consumo tra Lavoratori sede sociale in Monfalconese. Statuto sociale, Monfalcone, Stabilimento Tipografico Moderno, 1963 (annotato a penna). 
Italiana». All'art. 3 si creava una sezione di risparmio e si citava anche la figura del "consumatore non socio", al quale andavano rivolte delle attività di sensibilizzazione. Ora i soci potevano appartenere a qualsiasi mestiere (salvo quelli concorrenti) e dovevano essere residenti nel Friuli Venezia Giulia (FVG), che allora si costituiva in Regione autonoma. In un passaggio dell'art. 6 si obbligava i soci «ad acquistare presso gli spacci della Società il proprio fabbisogno di consumo in distribuzione presso i medesimi», anche se non venivano specificate le modalità di verifica e non erano previste delle sanzioni per eventuali violazioni (pare più una dichiarazione di volontà che un autentico obbligo). Attraverso alcune documentazioni conservate si può fotografare la rete degli 8 spacci operanti nel 1963: Monfalcone, Monfalcone-porto, Turriaco, Ronchi dei Legionari, San Canzian d'Isonzo, Pieris, Fiumicello e Papariano, con oltre 3.000 soci complessivi7. Lo storico Presidente del Consiglio di Amministrazione degli anni Sessanta e di buona parte dei Settanta è Silvestro Tonizzo. L'orientamento politico della CCLM è legato a quello del Partito Comunista Italiano (PCI). I negozi si configurano come spacci di limitate dimensioni (solitamente di 200$300 \mathrm{mq}$ ), in linea con quanto avveniva a livello nazionale, seguendo il motto "una cooperativa sotto ogni campanile», mutuato dalla nota linea togliattiana volta al radicamento del partito di massa («una sezione comunista e una Casa del Popolo sotto ogni campanile» ${ }^{8}$ ). Tuttavia, in quegli anni, iniziava per le cooperative di consumo «la lunga marcia delle unificazioni» e contemporaneamente l'aumento della superficie dei punti vendita (Renzo Testi). Proprio nel 1963 a Reggio Emilia si inaugurava il primo supermercato cooperativo di 1.400 mq di superficie di vendita, il Coop 1, organizzato su due piani ${ }^{9}$. Da lì prese avvio, ad esempio, il percorso di concentrazione delle cooperative di Reggio Emilia, prima su base comunale, poi intercomunale e successivamente provinciale. Nel 1974 costituirono la Coop Nordemilia, assieme a quelle di Parma, Mantova e Piacenza che avevano seguito itinerari similiio ${ }^{10}$ Questi processi di fusione furono appannaggio - per il momento - dell'avanguardia del

\footnotetext{
7 Cfr. A. CGIL, Lettura Record: n. file 312/1/6, Le funzioni della Cooperazione in difesa dei lavoratori: invito a spesarci nei nostri spacci!, Monfalcone, settembre-ottobre 1963. Gli spacci di Fiumicello e Papariano escono dal mandamento Monfalconese, ma insistono su un'area limitrofa. Fra il 1964 e il 1967, nella documentazione si ritrova anche lo spaccio di Cormons, il cui territorio rientra nell'area Goriziana. Cfr. A. CGIL, Lettura Record: nn. 312/1/7 e 312/1/12.

8 Intervista di Enrico Bullian a Renzo Testi, Reggio Emilia, 18 marzo 2014. Dopo la prima nota, il nome degli intervistati compare fra parentesi direttamente nel testo alla fine delle successive citazioni.

9 BOJARDI, Franco (a cura di), op. cit., p. 56. Si segnala che non fu la cooperazione di consumo a introdurre la moderna grande distribuzione in Italia, infatti il primo supermercato di Supermarkets Italiani aprì a Milano nel 1957. ZAMAGNI, Vera, BATTILANI, Patrizia, CASALI, Antonio, op. cit., pp. 344, 350.

${ }^{10}$ BOJARDI, Franco (a cura di), op. cit., p. 56.
} 
movimento cooperativo, che storicamente aveva le sue roccaforti nell'Italia centrale. Secondo Patrizia Battilani, a livello nazionale, «l'opera di modernizzazione procedette molto lentamente [...]: i negozi tradizionali restarono l'ossatura della cooperazione di consumo per tutti gli anni Sessanta» ${ }^{11}$. Ciò avvenne anche nel Monfalconese e proseguì allo stesso modo nella prima metà degli anni Settanta. I tempi non erano ancora maturi per un'autoriforma della rete di vendita, anche se esisteva la consapevolezza delle difficoltà nella gestione dei negozi. Il CdA della CCLM nel Bollettino d'informazione dell'aprile 1968 - nell'invito ai soci all'assemblea di approvazione del bilancio per l'esercizio 1967 - scriveva:

le vendite, purtroppo, non hanno avuto quello sviluppo che il Consiglio di Amministrazione ovviamente si aspettava: ciò è dovuto a diversi fattori. Enunciamo soltanto i principali, che possono così essere indicati:

- il perdurare della seria crisi del settore industriale, con il conseguente diminuito potere di acquisto dei lavoratori;

- la massiccia presenza delle grandi catene di distribuzione che attraverso i loro supermarket sviluppano una spregiudicata politica tesa a confondere il consumatore nelle sue scelte alimentari;

- l'attuarsi di una politica degli investimenti nel settore distributivo nel preminente interesse dei gruppi finanziari monopolistici;

- il perdurare di una ormai intollerabile pressione fiscale che colpisce soprattutto il Movimento Cooperativo ed i lavoratori a reddito fisso.

Nell'esercizio passato, la presenza e la funzione della nostra Cooperativa e del Movimento Cooperativo nel suo insieme, nell'azione contro il caro vita, la speculazione, le frodi, le sofisticazioni alimentari, in difesa del potere di acquisto dei lavoratori e della salute del consumatore, si è maggiormente sviluppata.

In particolare, le iniziative prese dal nostro Movimento si sono caratterizzate con l'affermazione e lo sviluppo del MARCHIO COOP e di tutti quei prodotti garantiti e controllati da COOP-ITALIA il grande CONSORZIO delle COOPERATIVE di CONSUMO ITALIANE ${ }^{12}$.

Da questa lettera ai soci si può concludere che non esisteva ancora un'autocritica sulla gestione della rete dei negozi, mentre i supermercati erano ancora considerati con sospetto, dal momento che svolgevano una «spregiudicata politica tesa a confondere il

${ }^{11}$ ZAMAGNI, Vera, BATTILANI, Patrizia, CASALI, Antonio, op. cit., p. 350.

12 A. CGIL, Lettura Record: n. file 312/1/13, Consiglio di Amministrazione della CCL sede Monfalcone (a cura di), Bollettino di informazione, Monfalcone, Stabilimento Tipografico Moderno, aprile 1968. 
consumatore nelle sue scelte alimentari». Sono stati riportati anche i passaggi promozionali finali, per dimostrare quali erano le parole d'ordine di allora, che, in diversi casi, attraverso un messaggio attualizzato rappresentano ancora oggi un patrimonio consolidato del movimento cooperativo.

Un altro passaggio interessante fa emergere l'impostazione di genere ancora imperante all'epoca. Si plaudeva alla nuova apertura della Coop a Ronchi dei Legionari, «negozio che corrisponde alle più moderne esigenze delle massaie e del consumatore» ${ }^{13}$, dove appare netta la sottesa separazione fra le donne, massaie massificate, e l'uomo, consumatore consapevole.

Immagini di lavoro e dell'inaugurazione del negozio ristrutturato n. 3 della CCLM, Turriaco 30 agosto 1967. Archivio del Consorzio Culturale del Monfalconese

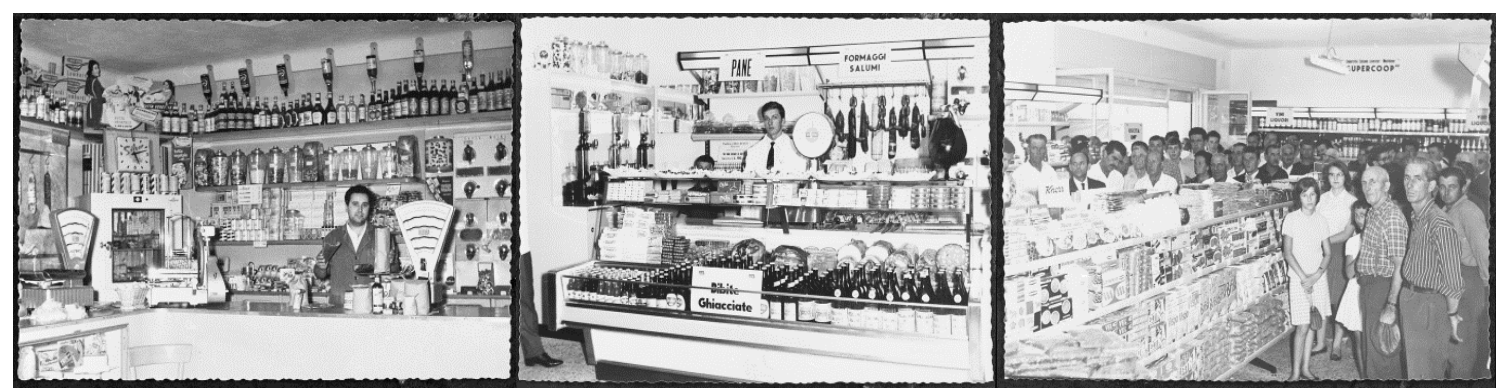

Nella Relazione del CdA dell'anno successivo, si può leggere come nel 1968 la CCLM abbia «contribuito alla creazione del nuovo grande magazzino COOP-ITALIA di Pordenone, che rifornirà le cooperative del Friuli-Venezia Giulia e del Veneto con una completa gamma merceologica», necessario per coordinare almeno una politica unitaria degli acquisti ${ }^{14}$. Nello stesso documento si comunicava dell'acquisizione di un terreno a San Canzian d'Isonzo «per la imminente costruzione di uno stabile da adibirsi a un modernissimo COOP»15. Lo sviluppo dei fatti è emblematico per dimostrare la prossimità (se non la sovrapposizione) che esisteva fra la CCLM e le organizzazioni del PCI. La "modernissima Coop" di San Canzian d'Isonzo fu inaugurata qualche anno dopo, il 29 maggio 1975, come si riportava addirittura in una cartolina dell'epoca.

\footnotetext{
13 Ibidem.

14 A. CGIL, Lettura Record: n. file 312/1/14, CCL - Soc. Coop. a r.l. Monfalcone, Relazione del Consiglio di Amministrazione sull'Esercizio Sociale chiuso il 31 dicembre 1968, Monfalcone, 27 aprile 1969.

15 Ibidem.
} 


\section{Casa del Popolo e Coop di San Canzian nel 1975. Archivio della Casa del Popolo di San} Canzian
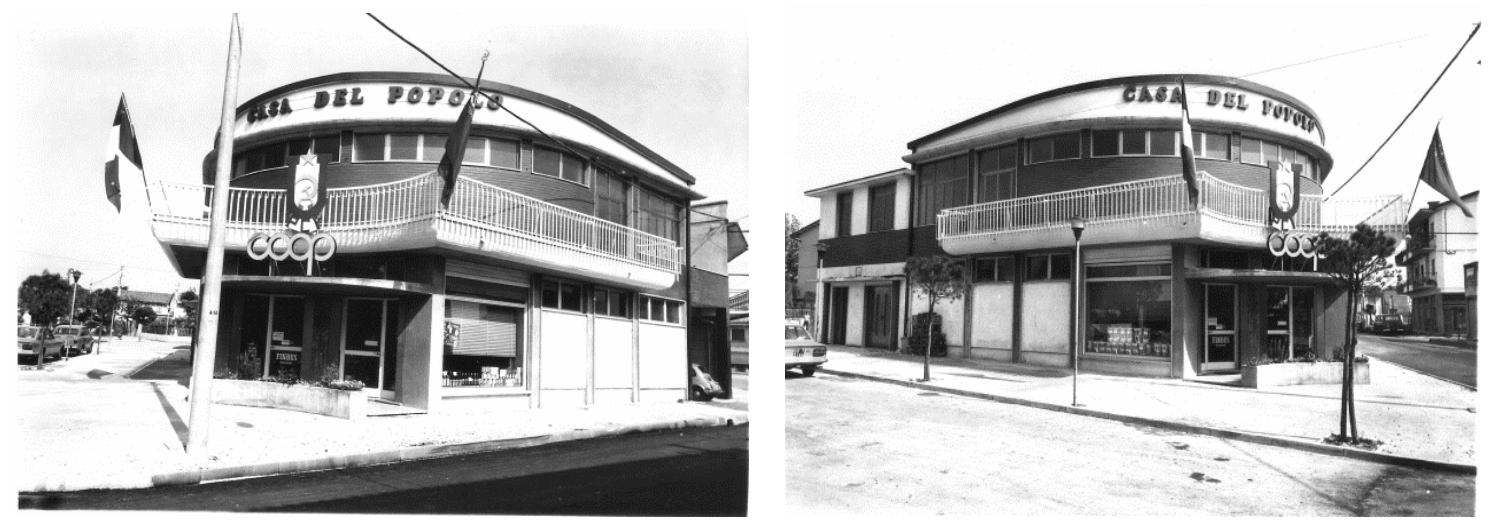

Le immagini dimostrano chiaramente la commistione fra le due strutture, la Casa del Popolo e la Coop, tanto da far svettare sopra la nota insegna di quest'ultima, la Falce e il Martello con la Stella. Il simbolo del lavoro era riferibile all’iconografia della sinistra e in particolare al Partito Comunista: evidentemente, ancora nel 1975, non veniva visto come un "limite" per il punto vendita la stretta correlazione fra le due simbologie, entrambe molto riconoscibili nell'immaginario collettivo. È opportuno rilevare che ciò avveniva in un Comune dove il PCI, da solo, godeva di una maggioranza assoluta di consensi, che gli permise di essere fortemente radicato sul territorio e di amministrare il Municipio lungo tutto il corso della Prima Repubblica. In altre parole, lungi dall'essere considerato un limite, in un siffatto contesto, l'associazione delle due simbologie poteva rappresentare un'opportunità, nonostante tali impostazioni cominciassero a modificarsi in contesti maggiormente "moderni”.

\section{La rivoluzione del Discount di Monfalcone nella seconda metà degli anni Settanta e la chiusura dei vecchi negozi}

Gli anni Settanta furono una stagione di grandi cambiamenti nella distribuzione commerciale a livello nazionale, come già evidenziato dalla storiografia. Nel 1973 Carrefour inaugurò a Carugate, in provincia di Milano, il primo centro commerciale con ipermercato in Italia, in grado di fatturare 21 miliardi di lire all'anno, mentre Standa, in collaborazione con la francese Euromarché, si avviava nella stessa direzione ${ }^{16}$.

${ }^{16}$ Il supermercato è una struttura con un'area di vendita al dettaglio che va dai $400 \mathrm{mq}$ ai $2.500 \mathrm{mq}$, mentre nell'ipermercato supera i $2.500 \mathrm{mq}$, arrivando anche a $12.000 \mathrm{mq}$. A differenziarli dal discount non è solamente l'estensione delle superfici, ma anche la filosofia di organizzazione degli acquisti, del lavoro e delle vendite, come si vedrà in seguito. 
Contemporaneamente erano comparsi i primi discount, che vengono così descritti dalla storica economica Patrizia Battilani: «strutture che non superavano i $500 \mathrm{~m}^{2}$, impostate principalmente sui non deperibili, che trattavano un numero ridotto di

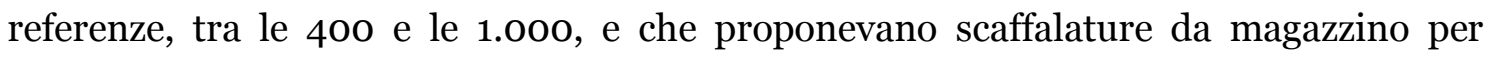
contenere i costi di manodopera» ${ }^{17}$. A metà degli anni Settanta, dunque, il movimento cooperativo nazionale si trovò davanti all'alternativa di introdurre uno dei nuovi modelli di negozio che andavano diffondendosi: i discount o gli ipermercati. Dopo alcuni anni di dibattito, Coop scelse inizialmente i primi. Fu una stagione intensa, caratterizzata da grande entusiasmo e risultati spesso positivi, come ricordato enfaticamente nelle testimonianze. Ciò è caratteristico delle fasi pionieristiche di fondazione di strutture innovative. Tuttavia i discount rappresentarono una parentesi di circa un decennio nel mondo della cooperazione di consumo, compresa fra la metà degli anni Settanta e la metà degli anni Ottanta. In seguito le Coop basarono la propria rete di punti vendita su ipermercati e supermercati. Le valutazioni che fornisce Battilani sono molto nette:

La crisi economica degli anni 1974-77 fece propendere il movimento cooperativo verso la formula discount, che ben presto avrebbe rivelato tutti i suoi limiti: il primo venne inaugurato a Pomigliano d'Arco nel febbraio 1976. Molteplici furono le ragioni della preferenza accordata ai discount invece che agli ipermercati: da una parte vi era la convinzione che la crisi avrebbe prodotto un effetto permanente sul modello di consumo, creando una forte domanda di prodotti non artificialmente abbelliti con pubblicità, promozioni o packaging accattivanti e che la formula del discount fosse quella più adatta a rispondere a questa esigenza; dall'altra vi era la necessità di trovare una risposta al fenomeno sempre più diffuso degli spacci aziendali voluti e gestiti dagli stessi operai in forma cooperativa; infine, aspetto non trascurabile l'investimento necessario era incomparabilmente minore.

«Noi dobbiamo avere il discount come alternativa, come risposta agli spacci aziendali. Non possiamo rispondere con dei no perché abbiamo visto che quando rispondiamo con dei no gli spacci aziendali sono stati fatti lo stesso ed allora dobbiamo rispondere con alternative valide» 18 .

${ }_{17}$ ZAMAGNI, Vera, BATTILANI, Patrizia, CASALI, Antonio, op. cit., p. 354.

${ }^{18}$ Intervento di CHECCUCCI, Fulvio, presidente dell'ANCC, Assemblea dei soci per l'anno 1975, in Coop Italia, 3-4 giugno 1976. 
Il discount venne ritenuto l'investimento più adatto per diversificare l'offerta della cooperazione di consumo e questo allontanò di un decennio l'ingresso nel comparto degli ipermercati ${ }^{19}$.

Lo storico Andrea Baravelli si focalizza in particolare sulla nuova generazione di dirigenti che subentrò nel movimento cooperativo a partire dagli anni Sessanta. Infatti, investendo gradualmente nella grande distribuzione, le Coop adottarono, in parte per i discount e soprattutto per i super e poi per gli ipermercati, «modelli di esercizio che imponevano di compiere - per i fortissimi investimenti, finanziari e di competenze, che richiedevano - un ulteriore scatto nel senso dell'adozione di una compiuta mentalità aziendale» ${ }^{20}$.

Fu infatti il nuovo gruppo dirigente, di formazione più spiccatamente manageriale rispetto alla precedente generazione, a puntare sul ruolo organizzatore dei consorzi provinciali e sul dispiegamento di un'indefessa opera di razionalizzazione. Con una storia a balzi, fatta di accelerazioni e rallentamenti, si sarebbe giunti al progressivo abbandono della concezione della cooperativa di consumo quale espressione della comunità locale, riuscendo ad allestire una rete di vendita moderna - centrata sui grandi supermercati e sul dislocamento strategico - e arrivando alla costruzione di una struttura reticolare del settore in grado di funzionare quale elemento di raccordo tra cooperative di consumo ed altri soggetti economici. Parallelamente, si sarebbe pian piano superato il grande limite del passato: l'esasperata competizione politica - alimentata dalle forti identità di parte - che nei decenni precedenti aveva invariabilmente inquinato ogni possibilità di sviluppo ${ }^{21}$.

Perché dunque soffermarsi sul caso del Discount di Monfalcone se in fondo rappresentò, in linea con quanto avveniva a livello nazionale, un'esperienza di breve durata? Perché sicuramente fu un fatto straordinariamente innovativo, che diede ottimi risultati a cavallo fra gli anni Settanta e Ottanta, permise di accrescere il numero dei cooperatori e dei soci e preparò le condizioni per le tappe e gli investimenti successivi.

19 ZAMAGNI, Vera, BATTILANI, Patrizia, CASALI, Antonio, op. cit., pp. 354-355. Va ricordato che dopo l'abbandono da parte delle Coop della formula dei discount a metà anni Ottanta, all'inizio del decennio successivo, sull'onda di una nuova crisi economica (1992-1993), si diffusero in Italia i discount di seconda generazione, portati da società soprattutto tedesche. Così, a metà anni Novanta, una parte della cooperazione investì nuovamente nella realizzazione di questi punti vendita e gli sforzi di varie Coop confluirono nel 1999 nella Di.Co spa, che nel 2002 contava 188 discount cooperativi. Tuttavia, recentemente, Coop è uscita dalla gestione dei Di.Co. Ibidem, pp. 362-363.

20 BARAVELLI, Andrea, Il giusto prezzo. Storia della cooperazione di consumo in area adriatica (1861-1974), Bologna, Il Mulino, 2008, p. 341.

${ }^{21}$ Ibidem, pp. 30-31. 
Ricostruito il contesto nazionale, ora si riporta quanto scriveva il già citato Bojardi nel 1994 in merito alla situazione Monfalconese.

Nei primi anni '70 in alcune fabbriche del Monfalconese nascono le esperienze degli spacci aziendali situati all'esterno degli stabilimenti e gestiti dai consigli di fabbrica (Nest Pack e Cartiera Timavo). In quest'opera vanno senz'altro ricordati i cooperatori che vi hanno lavorato in particolare il compianto Giorgio Fari.

Nel 1977 la cooperativa sulla spinta sindacale dell'Italcantieri e delle altre aziende del territorio, mediante CGIL-CISL-UIL e le ACLI [Confederazione Generale Italiana del Lavoro, Confederazione Italiana Sindacati dei Lavoratori, Unione Italiana del Lavoro e Associazioni Cristiane Lavoratori Italiani, ndA] si trasforma e si ristruttura concentrando la propria attività in un unico negozio discount a Monfalcone ove ancora oggi la Coop Consumatori ha il suo più grande supermercato della rete di vendita. Aperto per soli soci che svolge politica calmieratrice per volontà dei sindacati.

Tutti i piccoli negozi furono ceduti ai responsabili che li avevano sino ad allora gestiti per la Cooperativa.

La tipologia di vendita discount, oggi così prepotentemente ritornata sul mercato, fu allora provata a Monfalcone anche grazie ad una sperimentazione che il movimento cooperativo nazionale volle provare, inviando a collaborare con i dirigenti locali alcuni cooperatori emiliani e lombardi che seguirono i risultati altamente positivi raggiunti, in pochi mesi, dalla formula discount che aveva riscontrato l'alto gradimento del servizio e dei prezzi praticati ai soci-consumatori della cooperativa.

Tra il 1977 e il 1984 la cooperativa si sviluppa con una nuova generazione di supermercati a Villa Vicentina (UD), Ronchi dei Legionari e Gorizia [nella citazione andrebbero aggiunti i punti vendita di Torviscosa e Panzano, ndA] ${ }^{22}$.

Citata la storiografia, si ricostruisce tale periodo con documentazione e testimonianze più puntuali rispetto a quanto sopra riportato. A metà anni Settanta maturarono le condizioni per una radicale ristrutturazione della distribuzione cooperativa nel Monfalconese. Ci furono diversi fattori che permisero sostanzialmente di smantellare la rete di piccoli negozi per creare - inizialmente - un unico grande punto vendita, nella versione del discount (hard si direbbe oggi). I fattori alla base di questo percorso intrecciano inevitabilmente trend nazionali con aspetti più locali, la "grande" e la "piccola" storia che si intessono: i due principali elementi che consentirono questa virata furono da una parte l'avvento di una nuova generazione

${ }_{22}^{2}$ BOJARDI, Franco (a cura di), op. cit., p. 46. 
dentro il PCI e quindi negli organismi dirigenti delle Coop, più aperta a soluzioni innovative e concorrenziali; dall'altra l'intuizione di diversi Consigli di Fabbrica (CdF) di costituire degli spacci aziendali rivolti soprattutto alla tutela del potere d'acquisto dei lavoratori. Quanto si verificò nel Monfalconese corrispondeva alla fase nazionale espansiva del PCI, che non temeva il confronto e la contaminazione con altre forze politico-sindacali, accettandone il contributo anche all'interno di settori - come quelli della cooperazione rossa - che venivano considerati, fino a poco prima, di proprio esclusivo appannaggio. Questo si sovrapponeva alla fase di unità sindacale, che consentì - attraverso l'adesione degli organismi di rappresentanza - di orientare verso la cooperazione di consumo grandi masse di lavoratori, nonostante, almeno inizialmente, diverse iniziative di spacci aziendali fossero sorte dal basso in maniera spontanea, anche se successivamente alle mobilitazioni sindacali contro il caro vita. Infatti, negli anni Settanta, CGIL, CISL e UIL della provincia di Gorizia aprirono una vertenza per migliorare le condizioni in fabbrica ma anche "sul territorio", rivendicando servizi e alloggi sociali, trasporti, difesa del potere d'acquisto, strutture di medicina del lavoro $^{23}$. In altre parole, dentro la CCLM ci fu il passaggio da una struttura filo-PCI a una legata alla fase unitaria del sindacato.

Una prima avvisaglia del cambiamento è riscontrabile nell'intervento dello storico Presidente della CCLM, Tonizzo, al V Congresso nazionale dell'Associazione Nazionale delle Cooperative di Consumo (ANCC) tenuto nel 1974. Il documento è interessante perché permette di cogliere la fase di rinnovamento che stava iniziando a svilupparsi nel Monfalconese, con l'ingresso nel CdA della CCLM di rappresentanti delle organizzazioni territoriali dei lavoratori. Il Presidente Tonizzo - che non a caso lasciò l'incarico nel 1977 pur non essendosi opposto alle innovazioni - annunciava che

nell'ultima assemblea della nostra cooperativa i lavoratori sono entrati a far parte del Consiglio di Amministrazione per portare avanti assieme un programma di ristrutturazione della cooperativa stessa in modo da renderla strumento idoneo alle richieste provenienti dal mondo del lavoro che hanno trovato un positivo riscontro a livello delle Organizzazioni sindacali unitarie. Riteniamo che la richiesta unitaria delle Organizzazioni sindacali costituisca un importante fatto di maturazione

\footnotetext{
${ }^{23}$ Non era la prima volta che le Leghe delle cooperative affrontavano pubblicamente tali dibattiti e anche parte del movimento sindacale (CGIL) già negli anni Sessanta si era mobilitata a riguardo. Tuttavia con gli anni Settanta si modifica la portata di tali iniziative che divennero di massa, organizzate unitariamente da CGIL, CISL e UIL. Per cogliere la differenza fra le mobilitazioni degli anni Sessanta e Settanta, cfr. A. CGIL, Lettura Record: nn. file 312-1-5; 3123-1; 312-4-7; 312-4-10; 313-1-15; 98-1-15 e 106-3-6; manifesti nn. 235-236.
} 
perché testimonia l'impegno del Sindacato a livello unitario nell'affrontare i problemi esterni alla fabbrica ${ }^{24}$.

Si chiarisce che entrarono nel CdA i rappresentanti delle organizzazioni sindacali territoriali e non i lavoratori dipendenti della Cooperativa (non si trattava di una cogestione di modello tedesco). Inoltre, Tonizzo lucidamente ammetteva che, «per le condizioni particolari che hanno delle profonde radici storiche nel Friuli-Venezia Giulia il nostro Movimento [mandamentale e regionale] si trova in ritardo e deve superare l'attuale frazionamento», considerato che si procedeva «nel quadro del rafforzamento e dell'espansione del sistema nazionale di imprese cooperative» ${ }^{25}$.

Per ricostruire la fase "pionieristica" che portò da 8 piccoli negozi a un unico grande discount sono state raccolte, nel corso del 2014, diverse interviste ai promotori diretti di quella ristrutturazione. Questi testimoni sono i protagonisti del cambiamento, quelli che sostennero la necessità di tale scelta. Quindi sono i "vincitori” rispetto alla linea tradizionale degli spacci di limitate superfici e, pur nella diversità di opinioni che emerge in seguito fra i vari "riformatori", c'è una condivisione di fondo su molti punti di partenza. Relativamente a questa fase iniziale di sviluppo, sono stati intervistati in particolare Adriano Persi, Mario Dal Canto, Mile Marchese, Livio Aleotti e Lucio Pieri (quest'ultimo divenne un cooperatore qualche anno dopo rispetto agli altri) ${ }^{26}$. Ciascuno ha rivestito un ruolo diverso nella genesi del Discount di Monfalcone.

Ad esempio Persi - sindaco di Monfalcone negli anni Novanta - era un giovane militante del PCI, che era stato inserito nel CdA della CCLM dal Partito e che svolse una funzione importante nella ristrutturazione dei punti vendita. Persi è un testimone diretto dello scontro ideologico e generazionale che ci fu all'interno del $\mathrm{CdA}$, dove i "vecchi" non erano disponibili a modificare l'impostazione finora data alla CCLM: gli fu affidato - come lui stesso ricorda - il ruolo di "ariete" dentro il CdA, ma questo non gli pesò particolarmente perché «ti sentivi forte di queste idee nuove».

Quella generazione sicuramente appariva maggiormente aperta al confronto con il mercato e le sue regole, pur rimanendo in un quadro di forte spinta solidaristica e volontaristica. Affiorano spesso nelle memorie i racconti, legati soprattutto alla fase di

24 A. CGIL, Lettura Record: n. file 312/1/15, Intervento del Presidente della Cooperativa Consumo Lavoratori di Monfalcone comp. Silvestro Tonizzo al $V$ Congresso nazionale dell'Associazione Nazionale delle Cooperative di Consumo - tenutosi a Roma il 26-27-28 giugno 1974.

25 Ibidem.

${ }^{26}$ Interviste di Enrico Bullian a: Livio Aleotti, Reggio Emilia, 18 marzo 2014; Lucio Pieri, Ronchi dei Legionari, 31 marzo 2014; Mile Marchese, Ronchi dei Legionari, 31 marzo 2014; Mario Dal Canto, Fogliano-Redipuglia, 11 aprile 2014; Adriano Persi, Ronchi dei Legionari, 14 luglio 2014. 
immediato avvio del Discount, dei lavori volontari e gratuiti che venivano effettuati. Marchese era un giovane operaio della Nestpack che, finito il turno di lavoro, andava alla Coop a saldare le scaffalature e a predisporre il capannone per l'apertura del Discount. Non doveva essere infrequente nei primi anni di gestione del Discount vedere i componenti del CdA caricare la merce nelle scaffalature o spostare i pallets dal magazzino all'area vendita.

In generale, in tutti gli intervistati esisteva la chiara consapevolezza dei limiti della gestione precedente, considerata inefficiente e non più rispondente alle esigenze di consumo dei lavoratori. I gerenti dei negozi cooperativi, che occupavano al massimo 23 persone ciascuno, venivano quasi associati a una conduzione privatistica, caratterizzata alle volte da ammanchi. In risposta all'incapacità dei punti vendita delle Coop di essere convenienti, diversi CdF del Monfalconese costituirono degli spacci aziendali, che acquistavano della merce in blocco per poi rivenderla ai lavoratori quasi a prezzo d'acquisto. Non trascorse molto tempo per collegare queste esperienze dal basso dei CdF al magazzino di Coop Italia di Pordenone, che si premurò di soddisfare le richieste dei lavoratori. Il primo spaccio aziendale nacque nel Monfalconese attorno alla metà degli anni Settanta all'ex Solvay, la cui denominazione di allora era Nestpack. I promotori - impressi nella memoria collettiva - furono Giorgio Fari e i fratelli Dal Canto, Mario e Gino, tutti operai della stessa fabbrica. La formula era semplice ma dirompente: veniva comprata merce dai grossisti e rivenduta solo con il rincaro della fattura, con pochi articoli (Mario Dal Canto). Adriano Persi ricorda come l'azienda concesse l'utilizzo del vecchio edificio della pesa per adibirlo a piccolo spaccio. È opportuno citare che molti lavoratori della Nestpack provenivano da Rosignano Solvay (in Provincia di Livorno) e si erano stabiliti a Monfalcone in seguito all'acquisizione della Nestpack da parte della Solvay. Quest'ultima mantenne a Rosignano Solvay il ciclo della soda, mentre riconvertì la fabbrica di Monfalcone alla produzione di materie plastiche. Per far questo, una ventina di livornesi vennero mandati nel 1971 a un corso di riqualificazione professionale a Giussano (in provincia di Monza e della Brianza), dove esisteva già un impianto della Nestpack e nel 1972 furono trasferiti a Monfalcone (Mario Dal Canto). Molti dei testimoni originari del Monfalconese considerano utile l'esperienza dei livornesi che conoscevano Coop Proletaria, anche se Dal Canto - unico livornese intervistato - non conferma questa interpretazione, sostenendo piuttosto che ebbero molta fantasia e pragmatismo nell'inventare il Discount di Monfalcone. Ad ogni modo, dopo l'esperienza della Nestpack seguirono altre aziende e quindi nacque l'idea di avere un unico spaccio comune. Per questo alla fine del 1976 si formò nella Provincia di Gorizia un Comitato di Coordinamento per la Cooperazione, promosso da CGIL, 
CISL, UIL, Lega delle Cooperative (Federcoop), Confederazione Cooperative (Unione) e ACLI. In quegli anni si stabilì che la presidenza della CCLM veniva gestita con una rotazione biennale tra CGIL, UIL, CISL e ACLI, seguendo l'ordine esposto.

Il numero monografico «Speciale cooperazione» del novembre 1976 è dedicato proprio alla nascita del Comitato in preparazione della prossima apertura del Discount di Monfalcone. Nell'annuncio evidenziavano che quest'ultimo rappresentava la «seconda esperienza della Regione, dopo quella positiva avviata nel giugno 1976 presso le officine Danieli di Buttrio, dove attualmente si registrano vendite giornaliere per 4 milioni» ${ }^{27}$ e venivano sintetizzate le principali caratteristiche della struttura, con allegata la planimetria:

Il Comitato di Coordinamento per la Cooperazione, oggi è in grado di presentare concretamente una soluzione attraverso l'utilizzazione, come punto vendita discount, di un capannone di 700 metri quadrati con altri $2600 \mathrm{mq}$ di parcheggio, sito lungo la Statale 14 per Trieste, nell'immediata perifieria di Monfalcone, nei pressi della Zona industriale del Lisert ${ }^{28}$.

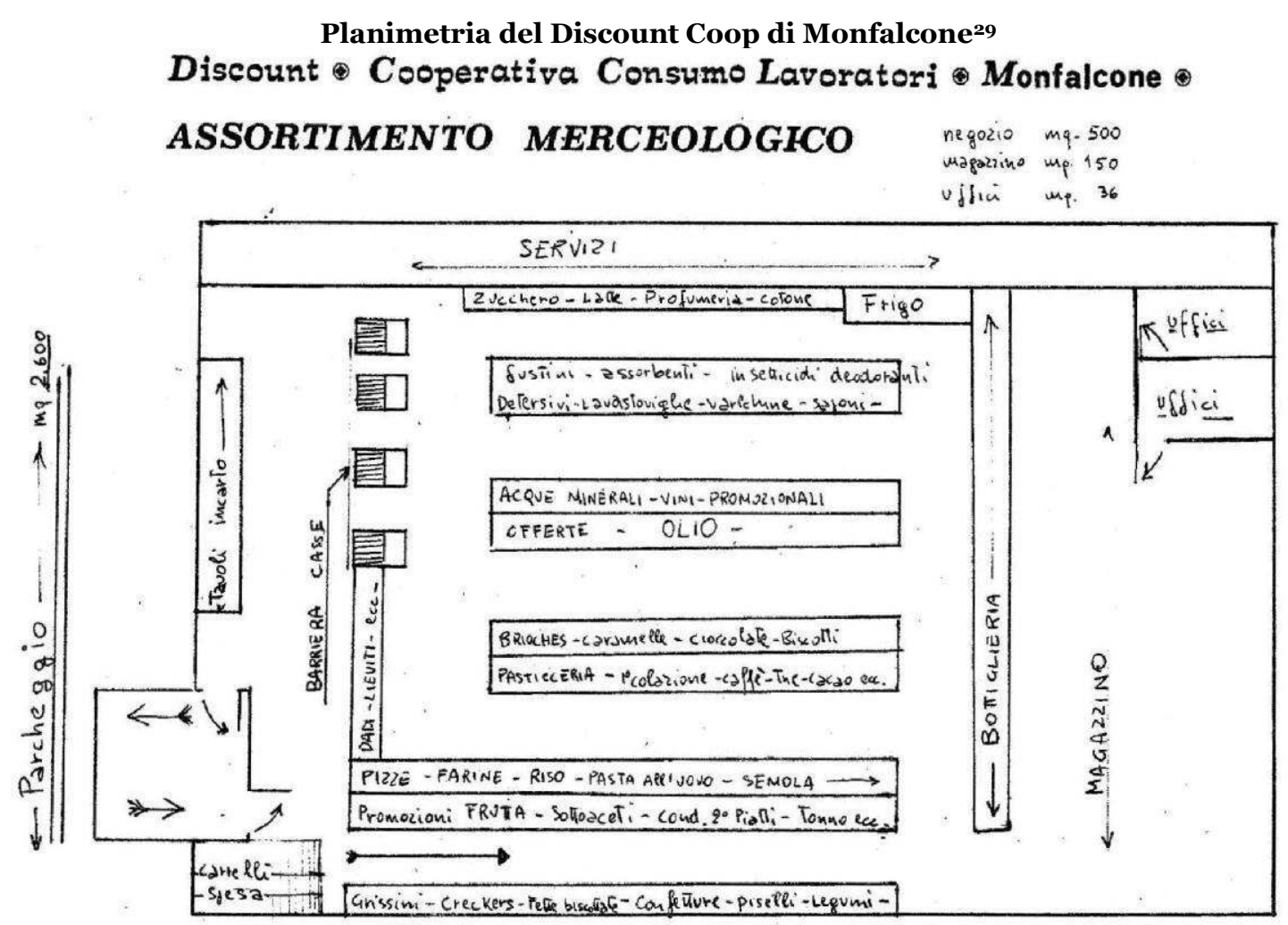

${ }^{27}$ A. CGIL, Lettura Record: n. file 312/1/18, Comitato di Coordinamento per la CooperazioneProvincia di Gorizia, in Speciale cooperazione, Monfalcone, stampato in proprio, novembre 1976.

${ }^{28}$ Ibidem.

29 Ibidem. 
Così, da questa pubblicazione del novembre 1976 si apprendono le tipologie merceologiche che proponeva il punto vendita (ad esempio non era ancora presente la carne) e si veniva a conoscenza della localizzazione. Anche questa risultava un'innovazione importante: la scelta strategica consisteva nel collocarsi immediatamente fuori dal centro cittadino, mettendo però a disposizione un'ampia area di parcheggio sulla Strada Stadale ad alta percorrenza in direzione Trieste.

L’individuazione dell'area del capannone di via Colombo è rimasta impressa a tutti i cooperatori di allora, anche perché seguirono lunghe prassi amministrative per ottenere la licenza. Fu Adriano Persi a "scoprire" il capannone, che era un magazzino di materiali edili: quello era il precedente utilizzo, considerato che, in quegli anni, la destinazione di tutta l'area - stando al Piano regolatore comunale - era industriale. Qui sotto si inseriscono delle immagini che mostrano come si presentava la struttura, di proprietà della Stignano, che fu affittata alla CCLM e che immediatamente avviò i lavori per consentire l'apertura del Discount.

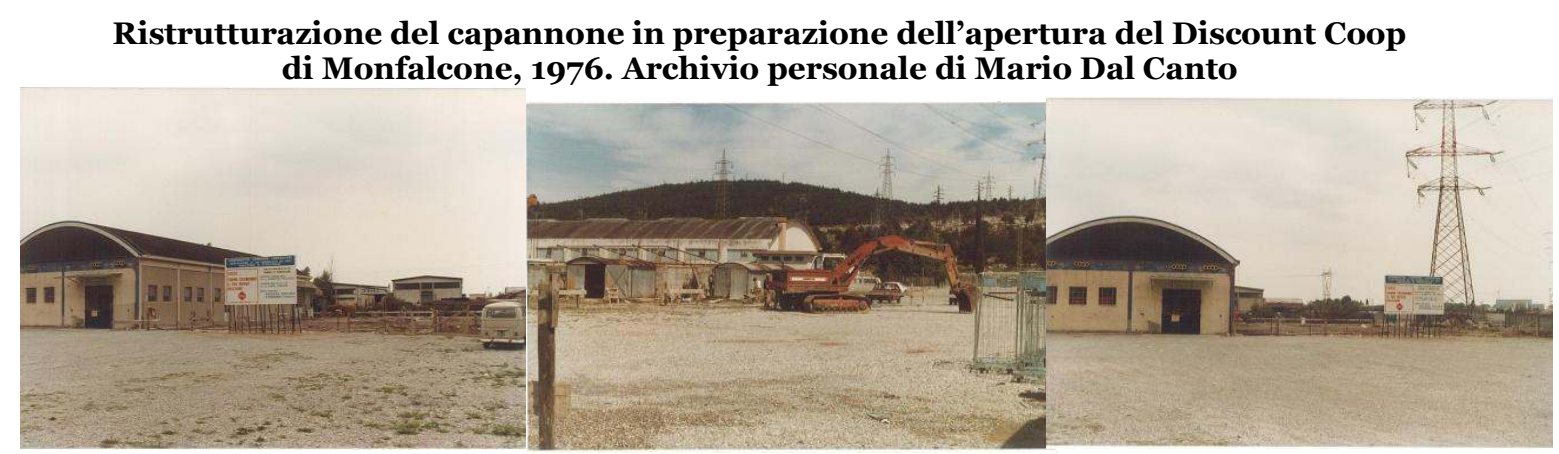

Come si nota, l'area si prestava per impostare un hard Discount, che facesse della concorrenzialità sul prezzo il proprio cavallo di battaglia, dal momento che si trattava di un capannone che si collocava in una zona industriale e che solo a seguito di un rifacimento completo avrebbe potuto eventualmente trasformarsi in supermercato.

La CCLM, effettuata la scelta di concentrare la vendita in un unico discount, procedette con la dismissione degli 8 piccoli negozi di paese, rivendendoli ai gerenti e lasciandoli la possibilità di affiliarsi a $\operatorname{Conad}^{30}$, con i quali fu raggiunto un accordo anche per trasferire una parte della ventina di dipendenti (Adriano Persi). Una minoranza di operatori restò invece alla CCLM, che iniziò l'attività con solo 7 addetti;

${ }^{30}$ Conad, acronimo di Consorzio Nazionale Dettaglianti, è una società cooperativa con sede centrale a Bologna attiva nella grande distribuzione organizzata. 
uno di questi fu Claudio Parolisi, che diventò in seguito capo negozio nel nuovo Discount di Monfalcone.

Il Discount Coop di Monfalcone aprì il 23 marzo 1977¹, mentre fu inaugurato il 4 giugno $1977^{32}$. Si riporta integralmente il volantino diffuso in quest'ultima giornata, anche per evidenziare l'impostazione che veniva data al punto vendita:

\section{Monfalcone - Via Colombo - SS 14 per Trieste}

\section{Un negozio radicalmente nuovo in difesa dei consumatori}

Il nuovo negozio di tipo "DISCOUNT" nasce dalla collaborazione tra le forze democratiche della Cooperazione, della Federazione unitaria CGIL-CISL-UIL e le ACLI, e dalla volontà e dall'impegno dei soci e dei dipendenti della COOPERATIVA CONSUMO LAVORATORI.

La nuova struttura di vendita è impostata con dei criteri assolutamente nuovi: infatti tutta una serie di costi di impianto e di servizio sono stati ridotti o eliminati: scaffalature più semplici, meno spazi frigoriferi, meno casse, migliore utilizzazione dello spazio.

L'assortimento è limitato ai prodotti essenziali, quelli che servono realmente a soddisfare oggettive necessità familiari. Si pensi che in un normale supermercato vi sono oltre 3000 prodotti: a Monfalcone, nel nuovo "DISCOUNT" COOP, le referenze sono circa 700, ma che rappresentano l'80\% dei consumi.

Un'offerta selezionata e vantaggiosa nei settori: pulizia della casa, igiene personale, scatolame, salumi, formaggi e latticini, ortofrutticoli stagionali, bevande, vini e liquori.

Gli articoli in vendita sono in gran maggioranza prodotti dalle Cooperative agricole o realizzati sotto il controllo della COOP ITALIA che garantisce con il proprio marchio la genuinità dei prodotti33.

La sede dell'assemblea dei soci, con il primo intervento riservato a Giorgio Fari come rappresentante del Comitato Unitario di Coordinamento per la Cooperazione, era - significativamente - la Sala della Federazione Unitaria Metalmeccanica. Lo slogan

\footnotetext{
${ }^{31}$ Atti del seminario Discount: nuova formula e struttura distributiva. Udine 1-2-3 marzo 1978, Bologna, Associazione Regionale Cooperative di Consumo Friuli Venezia Giulia, Lega nazionale cooperative e mutue, Coop Italia, 1978 (presunta), p. 47; A. CGIL, Lettura Record: n. file 312/1/20, Coop Discount Cooperativa Consumo Lavoratori Monfalcone, «Obiettivi raggiunti dal programma Coop 79: 1) 23 marzo 1977-23 marzo 1980 / tre anni al servizio dei consumatori associati».

${ }^{2}$ Archivio personale di Ezio de Luisa, San Canzian d'Isonzo, Cooperativa Consumo Lavoratori sede sociale Monfalconese, Volantino inaugurazione Discount Coop di Monfalcone e assemblea generale dei soci, 4 giugno 1977.

33 Ibidem.
} 
del volantino recitava: «Forme nuove di distribuzione nell'interesse del consumatore nella lotta al carovita» 34 .

In un comunicato ai soci diffuso 9 mesi dopo l'apertura in occasione della campagna promozionale per gli acquisti natalizi, si parla di 6.000 soci, che crescevano man mano si raccoglievano le adesioni dei CdF e in particolare dell'Italcantieri, di gran lunga la maggiore azienda del territorio, che, rimasta inizialmente esterna alla costituzione del Discount, poi assicurò la "massa" dei soci. Lo slogan scelto a chiusura del materiale pubblicitario era:

La Cooperativa Consumo Lavoratori non mira al profitto.

È un servizio sociale al consumatore.

Chi può dire altrettanto 35 ?

Già nel marzo 1978 fu organizzato a Udine da Associazione Regionale Cooperative di Consumo FVG, Lega nazionale cooperative e mutue e Coop Italia, un seminario sulla nuova formula e struttura distributiva dei discount. Nel convegno un intervento fu dedicato alla comparazione tra 10 discount cooperativi, tutti aperti fra il 1976 e il 197736. Da questa importante pubblicazione si possono trarre alcune informazioni significative per la ricerca. Quello di Monfalcone è l'unico discount a essere considerato "extra cittadino", mentre la maggioranza erano "cittadini", due "extra urbani di zona" (Buttrio e Gemona) e solo il discount di Fidenza risultava "aziendale". Per estensione delle superfici coperte, quello di Monfalcone (650 mq) era secondo solo a quello di Buttrio (1.000 mq). Per i parcheggi, Buttrio aveva $2.400 \mathrm{mq}$ e Monfalcone 2.000 mq, superati da Gemona con $4.450 \mathrm{mq}$. Da notare che ben 4 discount non possedevano parcheggi riservati, non trovandosi dunque in condizioni ottimali. Monfalcone, Buttrio e Fidenza erano i tre discount riservati solo ai soci, mentre gli altri possedevano la licenza di vendita al pubblico. A Monfalcone i locali erano ancora in affitto, per un

\footnotetext{
34 Ibidem.

35 Archivio personale di Ezio de Luisa, San Canzian d'Isonzo, Cooperativa Consumo Lavoratori sede sociale Monfalconese, Comunicato ai soci, 19 dicembre 1977.

${ }^{36}$ VALERIANI, Valerio, PERTOLDI, Alberto (a cura di), Elementi di comparazione tra alcuni discounts cooperativi, in Atti del seminario Discount, cit., pp. 42-54. Tutti i dati che seguono sono tratti dalle schede da p. 47 a p. 54. I discount cooperativi interessati dalla ricerca erano: Unicoop Firenze discount di Siena; Unicoop Pontedera discount di Pisa; A.C.M. Modena discount di Carpi; A.C.M. Modena discount di Modena; Coop Nord Emilia discount di Fidenza; Coop Operaie di Trieste discount di Trieste; Cooperativa di Monfalcone; Cooperativa di Buttrio; Cooperativa Carnica discount di Tolmezzo; Cooperativa Carnica discount di Gemona. La pubblicazione è interessante anche perché ripercorre l'evoluzione dei discount in Europa e approfondisce la situazione della Germania Federale e in particolare l'ALDI dei fratelli Albrecht. Sulla base del raffronto fra la situazione tedesca e quella italiana si ipotizzavano scenari futuri, riadattando le più avanzate esperienze europee al contesto socio-economico italiano.
} 
valore di 8.400.00o lire annue. A Monfalcone c'erano le scaffalature in ferro (solo a Trieste risultavano in legno), i frigoriferi murali e le celle frigorifere, ma non le isole frigo. Si utilizzavano i pallets, i transpallets e i muletti (solo a Trieste ciò non avveniva) e 4 registratori di cassa. Lo scontrino medio a Monfalcone (22.500 lire) era secondo solo a Buttrio (24.658 lire), mentre gli altri punti vendita variavano dalle 6.000 alle 13.00o lire. Monfalcone non si caratterizzava per un numero particolarmente alto di clienti per giornata d'apertura (211) rispetto ad altri punti vendita, come Trieste che raggiungeva anche gli 888 clienti, ma con spese medie molto più basse (6.461 lire). Per quanto riguarda la parte commerciale del punto vendita, a Monfalcone i prezzi erano praticati per 7 fasce di ricarico. Qui ogni discount si organizzava autonomamente, infatti in 4 casi non si adottava il metodo delle fasce di ricarico, mentre in altri casi le fasce erano minori ( 5 o $\quad 6$ ) e in altri arrivavano fino a 17 . Le referenze totali in assortimento a Monfalcone erano 688 (nella media rispetto agli altri discount), di cui 567 di generi vari (82\%), 81 di deperibili e semideperibili (12\%) e 40 non alimentari (6\%). Quest'ultima categoria allora era presente solamente in 3 discount. Le vendite nel 1977 ammontavano a Monfalcone a 1.097.984.00o lire, secondo solo al risultato di Pisa di 1.847.299.000 lire, che però era già stato aperto a fine 1976 e dunque non aveva perso i primi mesi dell'anno, come invece succedeva a Monfalcone. Se riferissimo le vendite all'intero anno, il gap si ridurrebbe e Monfalcone arriverebbe a 1.300.000.000 lire; tuttavia sarebbe superata - stando allo stesso ragionamento - da Trieste che giungerebbe a 1.776.000.00o lire. Ad ogni modo, tutti i discount riuscivano a garantire un utile. Monfalcone dichiarava un margine lordo di 8,7 per cento.

Anche questa pubblicazione dimostra come Coop Italia, a livello nazionale, fosse impegnata a sostenere iniziative sui discount (fino a poco prima assenti dal panorama nazionale) e a incentivare progetti pilota che all'epoca erano all'avanguardia. Non a caso, Coop Italia trasferì Livio Aleotti (che allora era un trentunenne assistente alle vendite nei supermercati cooperativi di Reggio Emilia) a Monfalcone per avviare il Discount, mentre Mario Dal Canto andò da Monfalcone al punto vendita cooperativo di Cavriago, in provincia di Reggio Emilia (Livio Aleotti; Mario Dal Canto). Sostanzialmente non esistevano esperienze nazionali da seguire e il metodo era molto innovativo; per questo Coop Italia favoriva uno scambio di conoscenze e delle migliori prassi.

Il ricercatore Baravelli, pur riferendosi all'«epoca pionieristica del movimento cooperativo» di fine Ottocento, parlò di distorsioni che riguardano la percezione sull'opera dei protagonisti di quella fase. 
I pionieri cooperatori vengono così usualmente rappresentati come persone fin dai primissimi esordi ben consapevoli di quali fossero i passi da compiere per assicurare un futuro di prosperità alla propria creatura associativa [...]. Per restituire valore a quegli stessi individui, invece, credo che occorra sottolineare il clima di incertezze, organizzative e di metodo, che segnarono gli esordi. Bisogna ricordare i successi ma anche i fallimenti, le incertezze e la perseveranza37.

La stessa valutazione è ascrivibile anche per i cooperatori degli anni Settanta, che con l'ottimismo della volontà e molta creatività affrontarono la ristrutturazione della rete di vendita, introducendo la formula del discount, che a Monfalcone rappresentò un autentico "nuovo inizio". Non a caso Lucio Pieri, Presidente della CCLM (1979-1981) e poi in Direzione organizzativa, ricorda che «la professionalità ce la siamo fatta sul campo».

La formula vincente nella gestione aziendale e commerciale del Discount di Monfalcone è rimasta impressa nella memoria di tutti gli intervistati. In sintesi, alcuni elementi chiave erano: i prezzi estremamente bassi; i (relativamente) pochi prodotti con merce spesso esposta direttamente negli scatoloni e/o lasciata sui pallets; i dipendenti disponibili a svolgere le mansioni e i lavori necessari («tutti i dipendenti facevano tutto», Mario Dal Canto); l'adesione alla filosofia "tutto vendita" tipica del discount, con riduzione al minimo delle aree destinate a magazzino e dei costi per scaffalature, impianti e strutture. Infatti, nei primi anni, il "magazzino" era ricavato perfino nel perimetro interno dell'area vendita, sopra le merci esposte (Mario Dal Canto). All'apertura del 1977, il Discount di Monfalcone non era fornito della gastronomia, del banco salumi e della macelleria, anche se di lì a poco si raggiunse un accordo con un commerciante locale, Dino Bergamasco, che forniva carne e salumi già confezionati. Anche l'ortofrutta inizialmente veniva venduta solo come prodotto confezionato (Mario Dal Canto). Il successo fu immediato, tanto che il punto vendita veniva identificato nell'immaginario collettivo più come il "Discount" che la "Coop" (Lucio Pieri). D’altra parte era il primo discount nella Provincia di Gorizia e uno dei primi a livello nazionale, quindi rappresentò una novità rilevante. Mario Dal Canto addirittura ricorda le corriere che venivano a rifornirsi periodicamente al Discount dall'Ungheria. Il Discount nel 1977 (e fino al 1983) non possedeva la licenza e quindi fu aperto esclusivamente per i soci, a differenza di quanto avveniva nei vecchi negozi di paese. In molti ricordano che Giorgio Fari, memore della sua esperienza

37 BARAVELLI, Andrea, op. cit., p. 26. 
nell'Associazione Ricreativa e Culturale Italiana (ARCI), preparò le tessere-socio di colore blu, mutuandole da quelle dell'ARCI (Mario Dal Canto; Adriano Persi).

Discount Coop di Monfalcone, seconda metà anni Settanta. Archivio del Consorzio Culturale del

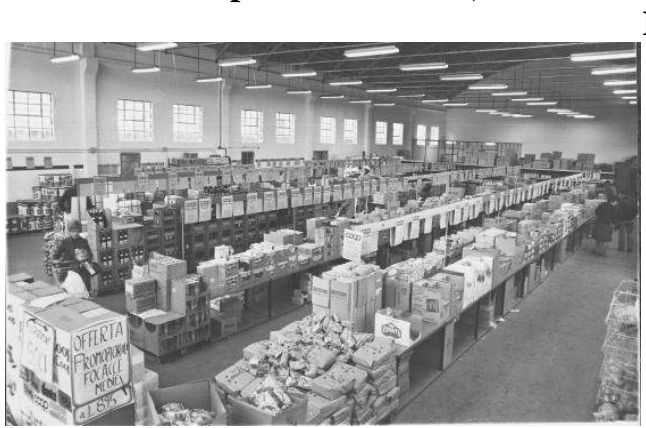
Monfalconese

Discount Coop di Monfalcone, seconda metà anni Settanta. Archivio personale di Mario Dal Canto
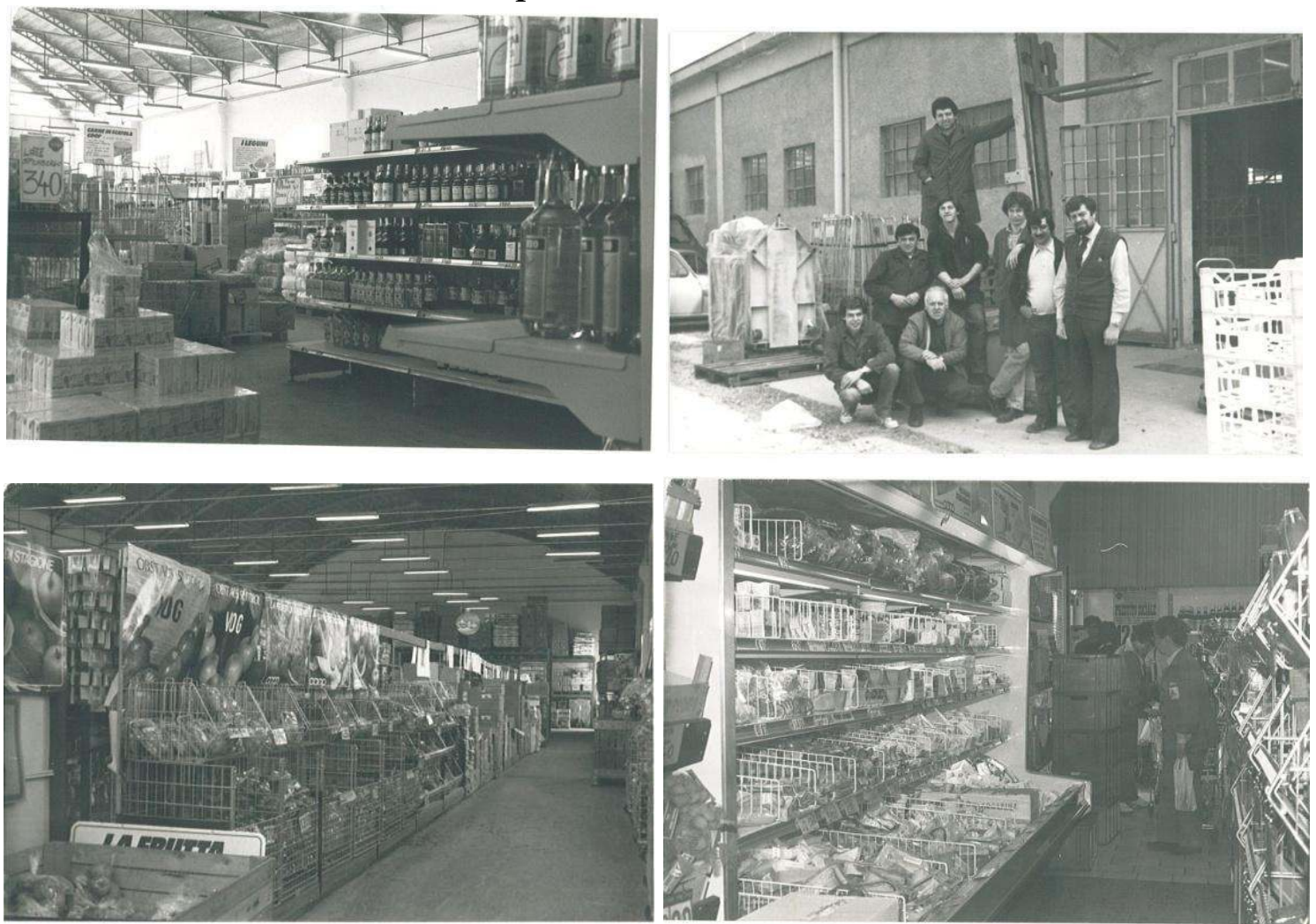

Le condizioni di lavoro nel primo periodo di attività del Discount di Monfalcone non dovevano essere ottimali, a causa delle carenze strutturali che solo diversi anni dopo (con la prima ristrutturazione-ampliamento del 1984) sarebbero state sanate. 


\section{L'ambiente di lavoro al Discount Coop di Monfalcone, seconda metà anni Settanta. Archivio personale di Mario Dal Canto}

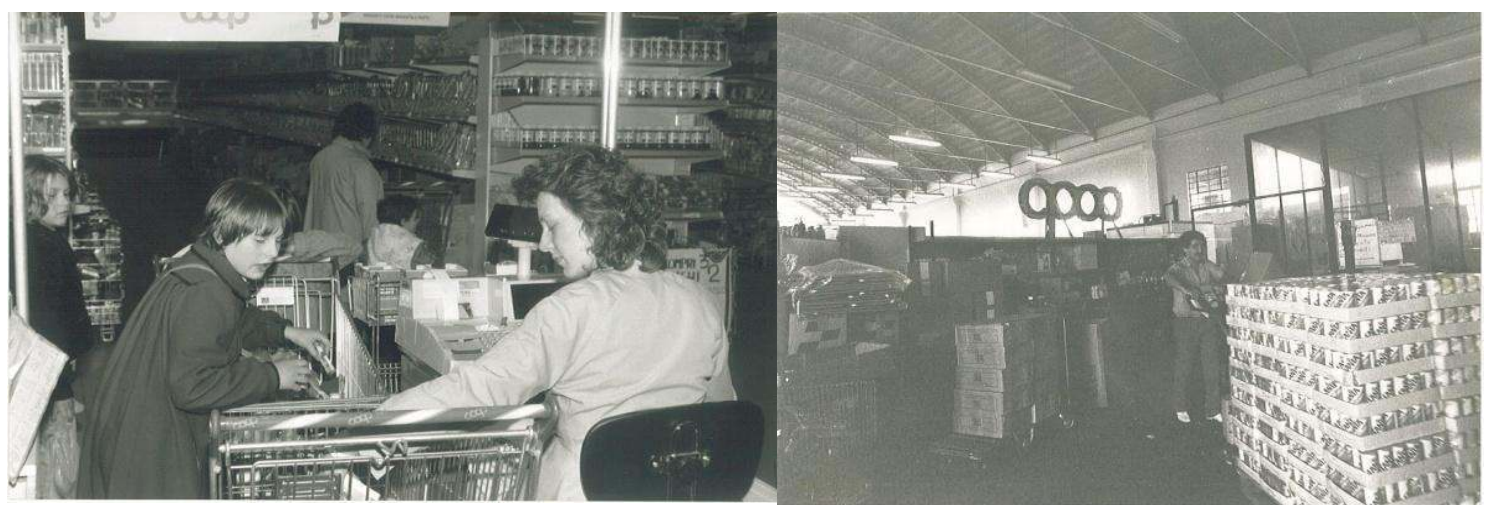

A titolo d'esempio, nell'immagine di sinistra si nota come operava allora la cassiera: non era ancora stato introdotto il banco per agevolare la raccolta dei prodotti, che spesso non erano ancora prezzati. Ciò significava che i prezzi dovevano essere memorizzati. Fra l'altro, i primi banchi erano leggermente inclinati per favorire il scivolamento degli articoli, mentre solo successivamente si diffusero con il tappeto scorrevole. Nell'immagine a destra si vede la movimentazione delle merci in magazzino, effettuata da Mario Dal Canto, che nei primi anni Ottanta era il Responsabile commerciale del Discount di Monfalcone. Ciò dimostra anche la commistione di lavori e mansioni che ogni cooperatore allora svolgeva. Il capannone era industriale, senza isolamento e controsoffittature, decisamente anomalo per una destinazione commerciale, almeno se considerata con i canoni attuali. Dal Canto ricorda come fino alla ristrutturazione-ampliamento del 1984 la luminosità fosse poca, mentre non c'era il riscaldamento in un capannone alto 9 metri. Non a caso le cassiere avevano le stuffette ai piedi in inverno e nelle giornate più rigide si provvedeva con un cannone rumoroso che sparava aria calda (di quelli utilizzati nell'edilizia, Lucio Pieri). Alla domanda sull'ambiente di lavoro di allora, Pieri rispose «allargo le braccia» e lo definì «ruspante», pur non segnalando problemi particolari di sicurezza; tutti i testimoni escludono casi di infortuni o malattie professionali invalidanti. Le condizioni di lavoro non apparivano massacranti, perché si movimentava una buona parte dei prodotti a bancale, mentre erano più pesanti a livello mentale (Mario Dal Canto). In quella fase iniziale si lavorava con passione - di fatto senza differenziazioni di livello - dove «tutti i dipendenti facevano tutto» (Mario Dal Canto).

Considerato che la scelta di aprire i discount cooperativi avvenne nella seconda metà dei "caldi" anni Settanta, si può intuire come quella decisione non fosse stata presa senza difficoltà all'interno del movimento. Non era solo la volontà di 
conservazione dell'impostazione originaria dei vecchi componenti dei CdA, ma esisteva anche un acceso dibattito attorno al "modello discount". Per ricostruirlo, si possono ripercorrere alcuni passaggi del già citato seminario del marzo 1978. Giorgio Riccioni, Vice Responsabile del Settore programmazione e sviluppo dell'ANCC, affermava che «il discount rappresenta oggi la formula più avanzata dell'organizzazione capitalistica del lavoro e dell'impresa e come tale, è compatibile soprattutto con un tasso di sviluppo generalizzato dei beni di consumo di massa»38. È ovvio che questo aprisse una discussione in particolare all'interno del movimento sindacale e dei partiti della sinistra. Secondo Riccioni ciò significava che i maggiori livelli di produttività erano

il frutto di una politica del lavoro che porta all'eccesso ogni forma di incentivo ed utilizza in maniera sfrenata il part-time. Per dare un'idea di questo fenomeno basti ricordare come in Germania fino al 30-35\% delle ore lavorative costituenti il budget di un punto di vendita sono gestite in assoluta libertà dal capo negozio. Ciò lascia facilmente supporre l'esistenza di un mercato del lavoro praticamente irripetibile, giustamente, nel nostro Paese39.

D’altra parte la politica del discount mirava alla minimizzazione dei costi attraverso la massimizzazione delle vendite, tendendo al soddisfacimento della clientela di ogni livello socio-economico. Per questo vi era la assoluta consapevolezza che «il discount offre il prezzo a scapito del servizio, della possibilità di scelta, della completezza dell'assortimento: tutti elementi, questi, presenti invece all'interno del supermercato»40. Inoltre, il limitato assortimento non consentiva grandi margini alle manovre sul prezzo di ogni prodotto: «Il discount è di per sé l'espressione più avanzata della stabilità del prezzo nel tempo in quanto ogni minima variazione influenza direttamente l'immagine del negozio e quindi le vendite»4. Infatti, un esperto del Despar del FVG, Piero Cattaruzzi, affermava che «La selezione dell'assortimento riduce al massimo, nel più breve tempo possibile, la decisione del consumatore che nel discount vede non un punto di scelta, ma un punto di approvigionamento» 42 . Cattaruzzi concludeva che

Senza eccezioni di sorta i prezzi di vendita dei prodotti devono essere inferiori del $10-15 \%$ rispetto a quelli praticati dalla migliore concorrenza presente sul mercato.

\footnotetext{
${ }^{8}$ Relazione di RICCIONI, Giorgio, op. cit., in Atti del seminario Discount, cit., p. 3.

39 Ibidem, p. 7.

40 Ibidem, p. 6.

${ }^{41}$ Ibidem, p. 8.

$4^{2}$ Relazione di CATTARUZZI, Piero, op. cit., in Atti del seminario Discount, cit., p. 11.
} 
[...] Azioni promozionali ed offerte speciali sono assolutamente evitate per non creare nel consumatore l'immagine che, in qualche modo, il punto vendita possa scendere al di sotto del prezzo normalmente praticato ${ }^{43}$.

Così, all'interno del mondo della cooperazione, ma anche in quello collegato politico-sindacale, si scelse il "modello discount". Ciò significa che fu considerato più determinante ridurre i prezzi rispetto al contrasto della maggiore flessibilità introdotta dai discount, almeno nella formula adottata originariamente nei Paesi europei più avanzati. In effetti, la versione italiana del discount fu rivista rispetto a quanto avveniva altrove, come ricordava Fulvio Riva nelle conclusioni del seminario. Se da una parte «Gli effetti più evidenti di questa razionalizzazione generale sono le cadute dei monopoli di posizione e il ridemensionamento del lucro, che viene ad assumere la più consona figura di giusta remunerazione della produttività»44, dall'altra «i discounts italiani hanno in maggioranza l'apparenza di superettes che sono state riprese in chiave di riduzione di assortimento, in chiave di risparmio di scaffalature, nonché di riduzione degli oneri del personale»45.

\footnotetext{
43 Ibidem, p. 14.

44 Conclusioni di RIVA, Fulvio, op. cit., in Atti del seminario Discount, cit.,p. 56.

45 Ibidem, p. 57.
} 


\section{* L'autore}

Enrico Bullian, classe 1983, ha conseguito il titolo di Dottore di Ricerca in Scienze Umanistiche indirizzo storico presso l'Università degli Studi di Trieste. Ha pubblicato monografie e saggi storici in particolare sull'emergenza amianto e sulle condizioni di lavoro nella cantieristica navale. Attualmente è borsista presso il Consorzio per l'AREA di ricerca scientifica e tecnologica di Trieste e in collaborazione con il Dipartimento di Studi Umanistici dell'Università degli Studi di Trieste, per la realizzazione di una ricerca sull'evoluzione delle condizioni di lavoro in alcune aziende regionali, fra le quali la Coop Consumatori Nordest. È Sindaco del Comune di Turriaco.

URL: < http://www.studistorici.com/progett/autori/\#Bullian >

\section{Per citare questo articolo:}

BULLIAN, Enrico, «La cooperazione di consumo nel Monfalconese nella seconda metà del Novecento. Dalla Cooperativa Consumo Lavoratori del Monfalconese alla Coop Consumatori Nordest», Diacronie. Studi di Storia Contemporanea : Il diritto miltante, 29/12/2014,

URL:< http://www.studistorici.com/2014/12/29/bullian_numero_20/ >

Diacronie Studi di Storia Contemporanea $\beta$ www.diacronie.it

Risorsa digitale indipendente a carattere storiografico. Uscita trimestrale. redazione.diacronie@hotmail.it

Comitato di redazione: Jacopo Bassi - Luca Bufarale - Elisa Grandi - Deborah Paci - Fausto Pietrancosta - Matteo Tomasoni - Luca Zuccolo

Diritti: gli articoli di Diacronie. Studi di Storia Contemporanea sono pubblicati sotto licenza Creative Commons 2.5. Possono essere riprodotti a patto di non modificarne i contenuti e di non usarli per fini commerciali. La citazione di estratti è comunque sempre autorizzata, nei limiti previsti dalla legge. 